\title{
2D/3D Characterization of AM IN625 For Calibrating and Validating AM Modelling Techniques
}

\author{
Michael G. Chapman ${ }^{1,2}$, Michael D. Uchic ${ }^{2}$, Michael A. Groeber ${ }^{2,3}$, Edwin J. Schwalbach², Sean P. \\ Donegan $^{2}$ and Marie E. Cox ${ }^{2}$ \\ 1. UES Inc. Beavercreek, OH, USA. \\ 2. Air Force Research Laboratory, Materials and Manufacturing Directorate, WPAFB, OH, USA. \\ 3. Ohio State University, Department of Integrated Systems Engineering, Columbus, OH, USA.
}

The Air Force Research Laboratory Additive Manufacturing Modelling Challenge Series has supplied the materials community with an assortment of advanced microstructural characterization of Laser Powder Bed Fusion AM IN625 [1]. The initial data release was designed to supply sufficient information to calibrate the various modelling techniques to address 4 different sets of challenges posed to the community. There are both micro and macro challenges for both the process-to-structure and the structure-to-properties predictions.

The micro-scale process-to-structure challenge required single-track characterization for a variety of different power and speed combinations of the laser. Top-down backscatter electron (BSE) montages (Figure 1a) were collected along with periodic cross-section BSE, Electron BackScatter Diffraction (EBSD), and etched optical images (Figure 1b,c,d) to obtain the shape characteristics of single-track depositions. Challenge participants were then asked to predict the shape characteristics of the tracks in various $2 \mathrm{D}$ structures at specific locations in the geometry. The $2 \mathrm{D}$ structures vary in shape, aspect ratio, and build orientation. The single-tracks and $2 \mathrm{D}$ structures were printed on top of a $5 \mathrm{~mm}$ thick printed pad of IN625 to simplify modeling, but this also complicated the characterization procedures.

The macro-scale structure-to-properties challenge supplied grain statistics (orientations and size distribution) of Hot Isostatic Pressing (HIP) + Heat Treated (HT) and non-HIP + HT tensile bar specimens that varied in thickness $(1 \mathrm{~mm}-5 \mathrm{~mm})$ and build direction relative to the tensile loading direction $\left(0^{\circ}-40^{\circ}\right)$. Replicate samples of each were also tested under room temperature and elevated temperature conditions $\left(1600^{\circ} \mathrm{F}\right)$. EBSD was performed on at least two orthogonal planes of each condition, at both corner and center locations of each specimen (Figure 2a, b), along with a BSE montage of the entire cross section to reveal grain and carbide microstructure across the sample (Figure 2c). Carbide statistics were calculated, which included a denuded zone near the surface of the sample. Optical montages were also collected to obtain surface roughness and pore statistics of each sample (Figure 2d). The challenge participants were asked to combine this information with data from ASTME8 test geometries of the same printed material to make predictions about the mechanical properties of the tensile bar population. Methods for measuring the grain statistics, including large systematic variance in a single sample, will be discussed.

The micro-scale structure-to-properties challenge consists of a single tensile bar with a $500 \mu \mathrm{m}$ square cross section, and asks for predictions about the evolving stress-state of selected grains. The specimen underwent High Energy Diffraction Microscopy (HEDM) (tomography, near field, and far field) at a synchrotron while the specimen was strained to a small plastic strain while at room temperature. HEDM experiments enable tracking the local average elastic strain within individual grains of a polycrystalline sample, however, the current method cannot accurately resolve the geometrical shape of the grains 
smaller than 5-10 $\mu \mathrm{m}$ in diameter. In order to provide an accurate representation of the local microstructure in 3D, the LEROY system at AFRL was used to perform a multimodal serial sectioning experiment [2]. Approximately 1000 sections at $1 \mu \mathrm{m}$ spacing were collected from the same region-ofinterest as the HEDM experiment. Each section has EBSD, BSE and optical data, and a 3D reconstruction of the first 580 sections and is shown in Figure 3[3].

\section{References:}

[1] M Groeber et al., JOM 70 (2018), p. 441

[2] M Uchic et al., Proc. $1^{\text {st }}$ Intl. Conf. on 3D Mat Sci (2012), p. 195

[3] M Groeber and M Jackson, IMMI 3 (2014).

[4] The authors acknowledge support for this work from the Air Force Research Laboratory. MGC acknowledges support through contract \#FA8650-15-D-5230.

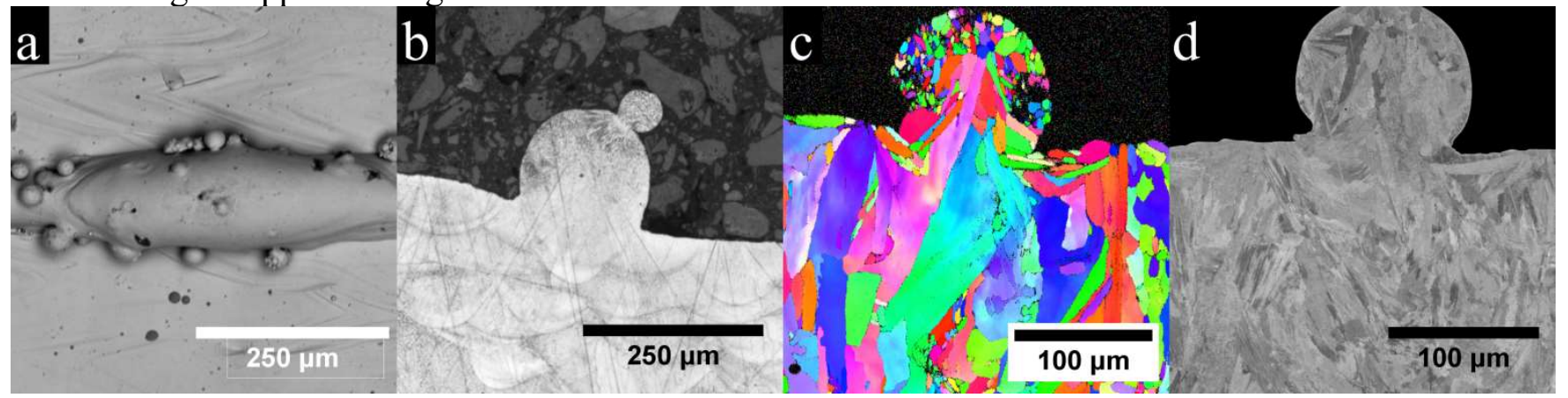

Figure 1. Examples of single track characterization a) Sub-region of top-down BSE montage, b) etched cross section optical image, c) cross section EBSD map, and d) cross section BSE image characterization.

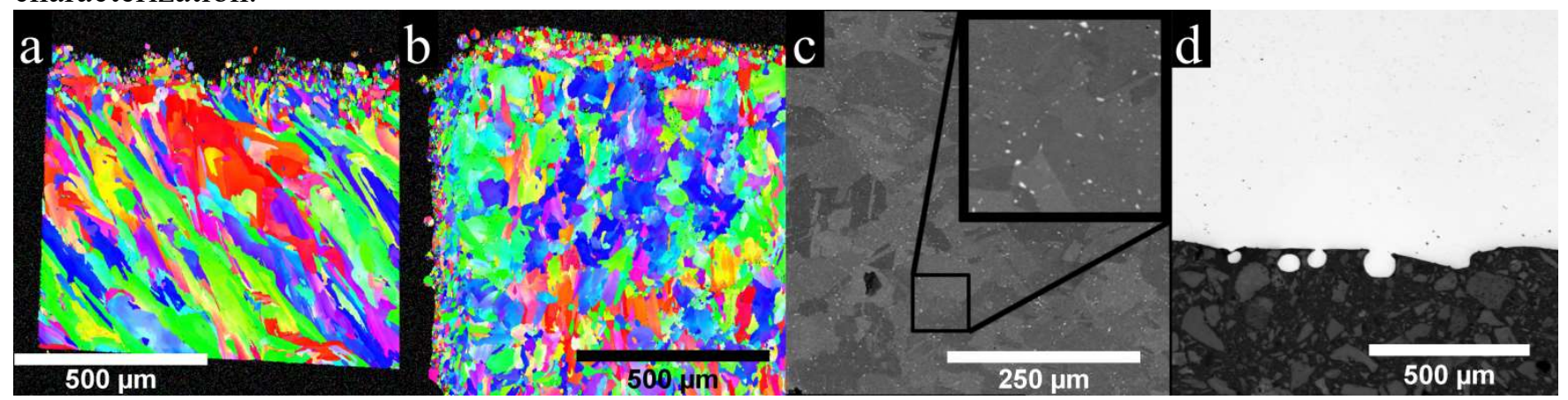

Figure 2. Orthogonal plane EBSD maps from a tensile specimen with the tensile axis $40^{\circ}$ from the build direction, a) perpendicular to the build direction and b) looking down the build direction. c) bse montage of a HIP+HT specimen with a magnified portion to highlight the carbide precipitates. d) subregion of an optical montage image for characterization of porosity and surface roughness.

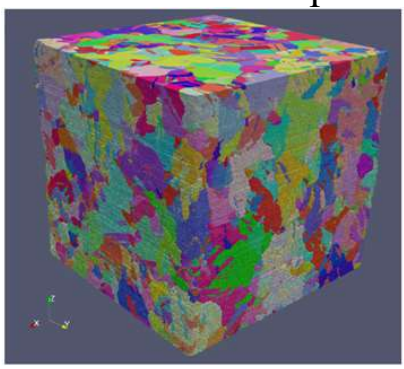

Figure 3. 3D reconstruction of 580 slices of serial sectioning data of a $500 \mu \mathrm{m}^{2}$ tensile bar. 